\title{
An Intermediate Service Composer Architecture for Dynamic Discovery and Invocation of Web Services from Mobile Devices
}

\author{
K.Kishoreanthuvan ${ }^{1,}$ S.Balaji ${ }^{2}$, S.Thamizharasan ${ }^{3}$, K.Kuzhandaivelu ${ }^{4}$ \\ ${ }^{I}$ (Assistant Professor, Department of computer Science \& Engineering, Christ College of Engineering and \\ Technology, Pondicherry, India) \\ ${ }^{2,3,4}$ (Department of computer Science \& Engineering, Christ College of Engineering and Technology, \\ Pondicherry, India)
}

\begin{abstract}
Web services describe a standardized way of integrating Web-based applications over an Internet protocol backbone. Mobile devices are getting more pervasive and it is becoming increasingly necessary to integrate web services into applications that run on these devices. We introduce a novel approach for dynamically invoking web service methods from mobile devices with minimal user intervention that only involves entering a search phrase and values for the method parameters. The architecture overcomes technical challenges that involve consuming discovered services dynamically by introducing a man-in-the middle (MIM) server that provides a web service whose responsibility is to discover needed services and build the client-side proxies at runtime. The architecture moves to the MIM server energy-consuming tasks. Such tasks involve communication with servers over the Internet, XML-parsing of files, and on-the-fly compilation of source code. The MIM Server programmed with the intelligence to identify a set of services whose collective functionality can serve the user's request. We perform extensive evaluations of the system performance to measure scalability as it relates to the capacity of the MIM server in handling mobile client requests, and device battery power savings resulting from delegating the service discovery tasks to the server.
\end{abstract}

Keywords: Geocode Services, Location Based Services (LBS), Man-in- the-Middle (MIM), Web services, XForms.

\section{INTRODUCTION}

A Web service is a method of communication between two electronic devices over the World Wide Web. The W3C defines a "Web service" as "a software system designed to support interoperable machine-tomachine interaction over a network. Man-in-the-middle (MIM) servers which will be used by mobile devices to discover needed web services and build their proxies. After getting the proxy, a mobile device can invoke a particular method of the web service and get the desired results. More specifically, the MIM server offers a web service which exposes a web method that the mobile device invokes and passes to it a search string. The MIM server's special service (or simply the MIM server) compares the submitted string to cached short-descriptions of Internet web services and generates a short list of services that best match the user's string.

To start with, the MIM server has a process that wakes up periodically to download a list of web service descriptions and associated URIs from a designated set of UDDI registries. More notably, the MIM server offers a web service that interfaces to three main processes which jointly fulfill the user's request. When using UDDI registries for service discovery, multiple costly network round trips are needed, and frequent unavailability of the wireless network may cause failures in the service discovery process, and will hinder the completion of the user request. . Many techniques have been used for dynamic discovery and invocation of web services from mobile devices and quite a number of tools have been in existence for the past few years. The major problem faced by the web services is, the user could not invoke composite web services and provide access of Multimedia, Data mining and Bio-Informatics. So a new architecture has been designed for composite web services and Multimedia in Mobile devices. It has a Location Based Service Framework which using Composite Web Services to access data in mobile devices.

\section{RELATED WORK}

Though, the particularities and restrictions of mobile devices and the mobile environment create great challenges for consuming web services [1]. However, the technology that allows devices to dynamically access web services is yet to established as there are infrastructural issues, which need to be tackled [2]. At first, several expensive network round trips are needed for service discovery while using UDDI registries and failures may cause in the service discovery process by frequent unavailability of the wireless network [1], and will delay the finishing point of the user request [3]. In [4] it was argued that the normal web service architecture which integrates a requestor, a broker, and a provider does not allow for designing a suitable service invocation on mobile devices. On the other hand, Halteren and Pawar [5] describe a platform for hosting web services on 
mobile devices by building on the Jini Surrogate Architecture Specification [6]. The DynWsLib library [7] is a .NET Framework-based technology that works by generating the client-side proxy class at runtime. Finally, we discuss the work in [8] which proposes a design for automatic generation of multimodal user interface from discovered WSDL files and a mechanism for service invocation. The approach relies heavily on the XForms technology [9] and requires the client browser to understand another markup language.

\section{PROPOSED WORK}

The proposed work provides a number of options for the user and provides a detailed output to the user using Intermediate based Architecture. This system operationally satisfies the needs what the user expects. The system designed is easily understandable by the end user. Location-based services are a general class of computer program-level services used to include specific controls for location and time data as control features in computer programs.

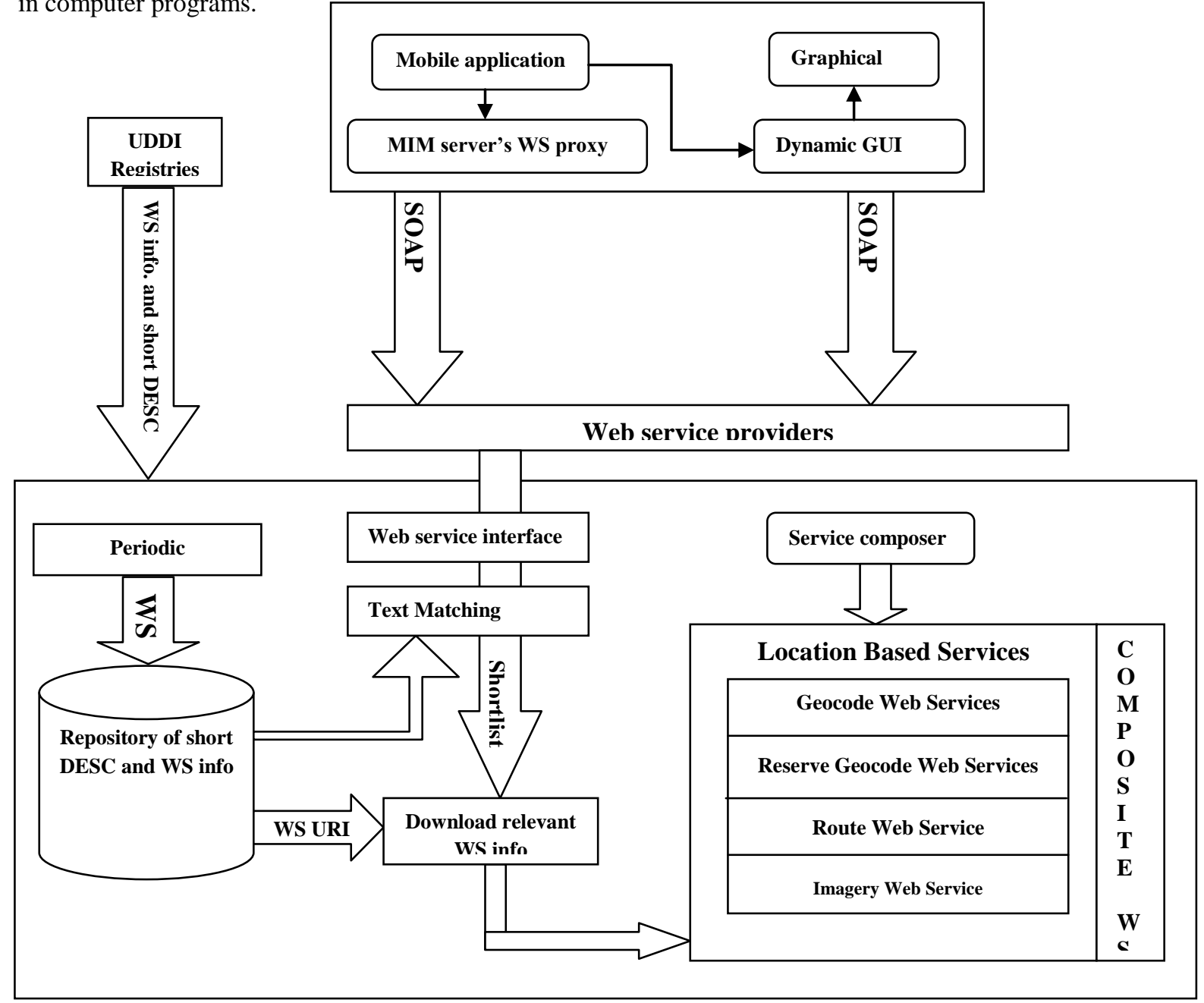

Fig.1: intermediate based architecture for dynamic invocation of web services

As such (LBS) is an information and has a number of uses in Social Networking today as an entertainment service, which is accessible with mobile devices through the mobile network and which uses information on the geographical position of the mobile device. This has become more and more important with the expansion of the Smartphone and tablet markets as well.

\subsection{Intermediate Based Server Architecture \\ 3.1.1 Boyer Moore Algorithm}

The Boyer-Moore exact pattern matching algorithm is probably the fastest known algorithm for searching text that does not require an index on the text being searched. This algorithm works by creating a "skip table" to each possible character. Using this skip table, the number of actual comparisons needed to locate a string can decrease, in some cases dramatically. 
The Boyer-Moore algorithm searches for occurrences of $\boldsymbol{P}$ in $\boldsymbol{T}$ by performing explicit character comparisons at different alignments. Instead of a brute-force search of all alignments (of which there are $\boldsymbol{m}-\boldsymbol{n}+$ 1), Boyer-Moore uses information gained by preprocessing $\boldsymbol{P}$ to skip as many alignments as possible.

The algorithm begins at alignment $\boldsymbol{k}=\boldsymbol{n}$, so the start of $\boldsymbol{P}$ is aligned with the start of $\boldsymbol{T}$. Characters in $\boldsymbol{P}$ and $\boldsymbol{T}$ are then compared starting at index $\boldsymbol{n}$ in $\boldsymbol{P}$ and $\boldsymbol{k}$ in $\boldsymbol{T}$, moving downward: the strings are matched from the end and toward the beginning of $\boldsymbol{P}$. The comparisons continue until either a mismatch occurs or the beginning of $\boldsymbol{P}$ is reached (which means there is a match), after which the alignment is shifted to the right according to the maximum value permitted by a number of rules. The comparisons are performed again at the new alignment, and the process repeats until the alignment is shifted past the end of $\boldsymbol{T}$. The shift rules are implemented as constant-time table lookups, using tables generated during the preprocessing of $\boldsymbol{P}$.

\subsubsection{WSDL File Reader}

The WSDL file downloader process is also multi-threaded, where in this case each thread maps to a WSDL file to download. For each considered file, this process checks for its existence in the cache, and downloads it from the Internet if a miss occurs. This process receives requests per second from the text matching process. It does not cause high-processing load, but rather a networking load that also affects the memory utilization.

The network can serve users at a rate of $\mathrm{N}$ users per seconds, where each user corresponds to an average of three WSDL files, each having an average size of S KB. Using a cache hit rate of $h$, each user request will effectively be downloading in KB. Now, assuming the network interface to the Internet has a bit rate of B kbps, we conclude that it can serve users per second.

Each thread will have a stack allocated, and dynamic memory whose size is equal to that of the file downloaded. Given that the execution code size is negligible, the memory usage of this process can be approximated. Generally when we use web service, at first we should add it in the web reference and then call its methods statically. Despite having a high speed, it isn't very flexible. In order to do it dynamically by dynamic invocation of web service, it has a very good flexibility.

\subsection{Mobile Application}

Location Based Service is to provide information about the location anytime and anywhere then to deliver the results of the replies from users via mobile devices. Since most of components in our framework are in the form of Web services, many Location Based applications can be easily developed as it is easy to reuse the components developed for other applications.

\subsubsection{Geocode Service Web Services}

Geocoding is the process of finding associated geographic coordinates (often expressed as latitude and longitude) from other geographic data, such as street addresses, or ZIP codes (postal codes). With geographic coordinates the features can be mapped and entered into Geographic Information Systems, or the coordinates can be embedded into media such as digital photographs via geotagging. A geocoder is a piece of software or a (web) service that helps in this process.

\subsubsection{Reverse Geocoding Web Service}

Reverse geocoding is the process of back (reverse) coding of a point location (latitude, longitude) to a readable address or place name. This permits the identification of nearby street addresses, places, and/or areal subdivisions such as neighborhoods, county, state, or country. Combined with geocoding and routing services, reverse geocoding is a critical component of mobile location-based services and Enhanced 911 to convert a coordinate obtained by GPS to a readable street address which is easier to understand by the end user. Public reverse geocoding services are becoming increasingly available through APIs and other web services as well as mobile phone applications. These services require manual input of a coordinate, capture from a GPS, or selection of a point on an interactive map; to look up a street address or neighboring places.

\subsubsection{Route Web Service}

Routing web service, which provides highly accurate point to point routing and route re-calculation using web services. Routing web services deliver turn-by-turn directions across multiple devices and platforms. With this flexible, easy to use web service you can:

- Quickly and easily integrate vehicle, bicycle or pedestrian routing into your existing web, mobile and desktop applications

- Build navigation applications that provide real-time, turn-by-turn routing to your users

- Integrate with other services to create rich interactive experience on the web, iPhone, Symbian, J2ME and beyond. 
Routing service is the only web-service that allows you to build applications that deliver real-time, turn-by-turn routing across a global database that includes roads, cycle-ways and footpaths. Whether you are developing for web, desktop or mobile, our fast, reliable Routing service helps you build applications that will surpass your user's expectations.

\subsubsection{Imagery Web Service}

Imagery Services is a set of programmable SOAP services that allow you to match addresses to the map, search for points of interest, integrate maps and imagery, return driving directions, and incorporate other location intelligence into your Web application.

\section{CONCLUSION}

Our design provides web service discovery services to personal applications running on mobile devices, where composite services can be used to extend the functionality of such applications. However, the applications from accessing composite web services that performs computationally intensive tasks, as in bioinformatics, data mining, and multimedia processing. But, since web service entities are usually autonomous and heterogeneous, how to connect and coordinate them is a challenging task that is not suited for mobile devices. So, the Intermediate Based Server can be programmed with the intelligence to identify a set of services whose collective functionality can serve the user's request. In fact, the Intermediate Based Server is well suited to coordinate the functions of such services and provide an interface to the mobile device with the architecture.

\section{REFERENCES}

[1] Q. Yu, X. Liu, A. Bouguettaya, and B. Medjahed, Deploying and Managing Web Services: Issues, Solutions, and Directions, The Very Large Databases J., (Springer) vol. 17, no. 3, pp. 537-572, 2008.

[2] Hassan Artail, Kassem Fawaz, Ali Ghandour, A Proxy-Based Architecture for Dynamic Discovery and Invocation of Web Services from Mobile Devices IEEE Trans. Services Computing, Vol. 5, No. 1, January-March 2012

[3] Q. Sheng, B. Benatallah, Z. Maamar, and A. Ngu, Configurable Composition and Adaptive Provisioning of Web Services, IEEE Trans. Services Computing, vol. 2, no. 1, pp. 34-49, Jan.-Mar. 2009.

[4] L. Li, M. Li, and X. Cui, The Study on Mobile Phone-Oriented Application Integration Technology of Web Services, Proc. Int'1 Conf. Grid and Cooperative Computing (GCC), Apr. 2004.

[5] A. Halteren and P. Pawar, Mobile Service Platform: A Middleware for Nomadic Mobile Service Provisioning, Proc. IEEE International Conf. Wireless and Mobile Computing, Networking and comm... (WIMOB), 2006.

[6] Nicolas Guelfi, Egidio Astesiano, Gianna Reggio, Scientific Engineering of Distributed Java Applications (Third international Workshop, FIDJI 2003 Luxembourg-Kirchberg, Luxembourg, November 2003

[7] C. Wever, DynWsLib Tutorial, http://www.thinktecture.com/resources/software/ DynWsLib/default.html, 2011.

[8] R. Steele, K. Khankan, and T. Dillon, Mobile Web Services Discovery and Invocation through Auto-Generation of Abstract Multimodal Interface, Proc International Conf. Information Technology: Coding and computing (ITCC'05), vol.2, pp. 35-41, 2005.

[9] T.V. Raman, XForms: XML Powered Web Forms [ISBN: 0321154991]. 